\title{
Combined Effects of Surface Morphology and Mechanical Straining Magnitudes on the Differentiation of Mesenchymal Stem Cells without Using Biochemical Reagents
}

\author{
Ji-Yeon Jang, ${ }^{1}$ Shi Woo Lee, ${ }^{2}$ So Hee Park, ${ }^{3}$ Ji Won Shin, ${ }^{3}$ ChiWoong Mun, ${ }^{3}$ Su-Hyang Kim, ${ }^{3}$ \\ Dong Hwa Kim, ${ }^{3}$ and Jung-Woog Shin ${ }^{3,4}$ \\ ${ }^{1}$ RঔD Center, Genewel Co., Ltd., Sangdaewon 1-dong, Jungwon-gu, Seongnam, Gyeonggi-do 462-724, Republic of Korea \\ ${ }^{2}$ R\&D Center, Genewel Co., Ltd., Baecto-ri, Hyangnam-eup, Hwaseong, Gyeonggi-do 445-924, Republic of Korea \\ ${ }^{3}$ Department of Biomedical Engineering, Inje University, Gimhae, Gyeongnam 621-749, Republic of Korea \\ ${ }^{4}$ Cardiovascular and Metabolic Disease Center, FIRST Research Group, Institute of Aged Life Redesign, Inje University, Gimhae, \\ Gyeongnam 621-749, Republic of Korea \\ Correspondence should be addressed to Jung-Woog Shin, sjw@bme.inje.ac.kr
}

Received 22 August 2010; Revised 19 December 2010; Accepted 11 January 2011

Academic Editor: George E. Plopper

Copyright (๑) 2011 Ji-Yeon Jang et al. This is an open access article distributed under the Creative Commons Attribution License, which permits unrestricted use, distribution, and reproduction in any medium, provided the original work is properly cited.

Existing studies examining the control of mesenchymal stem cell (MSC) differentiation into desired cell types have used a variety of biochemical reagents such as growth factors despite possible side effects. Recently, the roles of biomimetic microphysical environments have drawn much attention in this field. We studied MSC differentiation and changes in gene expression in relation to osteoblast-like cell and smooth muscle-like cell type resulting from various microphysical environments, including differing magnitudes of tensile strain and substrate geometries for 8 days. In addition, we also investigated the residual effects of those selected microphysical environment factors on the differentiation by ceasing those factors for 3 days. The results of this study showed the effects of the strain magnitudes and surface geometries. However, the genes which are related to the same cell type showed different responses depending on the changes in strain magnitude and surface geometry. Also, different responses were observed three days after the straining was stopped. These data confirm that controlling microenvironments so that they mimic those in vivo contributes to the differentiation of MSCs into specific cell types. And duration of straining engagement was also found to play important roles along with surface geometry.

\section{Introduction}

Mesenchymal stem cells (MSCs) can differentiate into a variety of cell types such as osteoblasts, adipocytes, chondrocytes, ligament cells, and smooth muscle cells [1-3]. It is thought that MSC transplantation is safer than traditional embryonic stem cell therapies. For example, Barry and Murphy [1] reported that a recipient's immune response, measured by the recipient T-cell activity, was significantly subdued when MSCs were used instead of traditional stem cells. The plasticity and relative safety of immune reactions make MSCs attractive candidates for therapeutic research and applications.

Most research on the control of MSC differentiation into desired cell types uses a variety of biochemical reagents such as growth factors $[4,5]$, even though the over-expression of growth factors is associated with possible side effects such as the acceleration of cancer metastasis [6]. In addition to the effects of growth factors, the effect of substrate stiffness, that is, purely physical effect, has also been reported. For example, MSCs were found to differentiate into progenitors of neuron-, muscle-, or osteo-related cells depending on substrate stiffness without the use of any growth factors [7]. Thus, biochemical reagents such as growth factors as well as the physical environment play roles in the differentiation of stem cells. Ideally, the physical environment should be identical to that which the cells experience in the human body.

Given that substrate properties affect MSC differentiation, mechanical stress must also affect differentiation. Most cells are continuously subject to physical stresses that may 
alter the external environment and ultimately play a role in cell proliferation and differentiation. For example, muscle cells acquire specific properties such as their shape from experiencing repeated tensile stretching while being held in a substrate geometry constructed by fibers. Therefore, studies on the effects of mechanical stimuli on cells are ongoing [811]. Mechanical stimuli can be classified into three types: compression, tension, and shearing. Here, we have focused on the effects of tensile stimuli on MSCs.

Many researchers have reported the effect of tensile stimuli on the fate of MSCs [11-15]. Chen et al. [12] examined the effects of two different strain magnitudes, $3 \%$ and $10 \%$, on MSC. The expression of osteo-related genes was found after 8 hours of continuous stimulus under the 3\% strain, but it did not last long. The expression of genes related to ligament or tendon cells was not observed under these conditions. Under the $10 \%$ strain condition, no significant expression of osteo-related genes was found. After 48 hours of $10 \%$ strain, however, the cells expressed ligament/tendonrelated genes, and this lasted for a sustained period. These results suggest that the magnitude of strain can direct MSC differentiation. Other reports have suggested that continuous $10 \%$ uniaxial straining for a day promoted the expression of genes related to smooth muscle cells (SMCs) when MSCs were cultured on smooth surfaces [14]. However, the expression diminished when the stimulation stopped. This was explained by the rearrangement of cells, a general cellular response for resisting external stimulation and/or excitation.

Cells attached but not fixed to a substrate try to reorient themselves in the direction perpendicular to the direction of tensile strain. After cells reorient during the course of an experiment, however, testing for any further effects of tensile stress loses significance because in real tissues, cell rotation is hampered by physical environment. For example, the cells within ligaments and muscles are constantly subject to uniaxial strain, yet these cells remain parallel to the stress due to the unidirectional bundles of fibers that prevent reorientation. Thus, physical environment plays a role in cell function, and the prevention of cell rotation maximizes an individual cell's activity [16]. Therefore, to accurately model biological conditions, the strain on cells as well as the cell orientation must be kept constant. Consequently, we studied MSC differentiation and changes at the gene expression level resulting from different magnitudes of tensile strain and substrate geometry under a consistent cellular environment. Also we investigated how long those factors affect the differentiation of MSCs into a cell type.

\section{Materials and Methods}

2.1. Fabrication of Microgrooved Substrates. A basement plate (wafer) of silicon was made using photolithography and deep reactive ion etching (DRIE). The substrate material where cells were to be seeded was made according to the manufacturer's protocol (Sylgard 184 silicone elastomer kit; Dow Corning, Midland, MI, USA), spin-coated, and cured at $125^{\circ} \mathrm{C}$ for $20 \mathrm{~min}$. Each groove was confirmed to be $20 \mu \mathrm{m}$ and $3 \mu \mathrm{m}$ for width and depth, respectively (Figure 1). A substrate without grooves was also made for use as a control.
2.2. Preparation and Seeding of MSCs. MSCs were isolated from the femur and tibia of New Zealand white rabbits as described elsewhere [17] and cultured in Dulbecco's Modified Eagle Medium with low glucose (DMEM-LG, Gibco, Grand Island, NY, USA) supplemented with fetal bovine serum (10\%; FBS, Gibco) and penicillin-streptomycin solution (1\%; P/S, Hyclone, Logan, UT, USA). Cells were kept at $37^{\circ} \mathrm{C}$ in a humidified incubator containing $5 \% \mathrm{CO}_{2}$. The substrates were cut into $0.5 \mathrm{~cm} \times 3 \mathrm{~cm}$ pieces, washed with deionized water, and autoclaved for cell seeding. The surfaces were treated by plasma (APP Co., Ltd, Suwon, Korea) and coated with fibronectin $(15 \mu \mathrm{L} / \mathrm{ml}$, Sigma, St. Louis, MO, USA). Finally, the cells were seeded onto the surface at a density of $2 \times 10^{4}$ cells $/ \mathrm{cm}^{2}$.

2.3. Application of Uniaxial Strain. To apply uniaxial strain to MSCs, a modified Flexcell plate (TT-4001U; uniaxial stretch unit, Flexcell International Corp., McKeesport, PA, USA) was used. Specifically, 48 hours after seeding the MSCs, the substrates were attached to Flexcell 6-well plates, and cyclic uniaxial strain was applied for 3 days. Cyclic uniaxial strain was administered continuously at a frequency of $0.26 \mathrm{~Hz}$ at magnitudes of $3 \%$ and $10 \%$. The cells were harvested three times: immediately before and after straining and 3 days after straining stopped.

2.4. DNA Content. To study the proliferation of MSCs, the number of cells was determined by measuring DNA content using Quant-iT PicoGreen dsDNA Reagent and Kits (Molecular Probes, Eugene, OR, USA). Briefly, cell membranes were permeabilized by triton X-100 and QuantiT PicoGreen dsDNA Reagent which is an ultra sensitive fluorescent nucleic acid stain, was used for quantitating double-stranded DNA in cells. Finally, the samples were analyzed using Multi-Detection Microplate Reader (Synergy HT; Bio-Tek, Winooski, VT, USA).

2.5. Staining of Actin Filaments. To observe the cytoskeletal arrangements of MSCs, actin filament orientation was observed using rhodamine phalloidin (Molecular Probes). The cells were fixed in $10 \%$ formalin solution, neutral buffered (10\%, Sigma, Cat.\# HT501128), permeabilized with $0.1 \%$ Triton $\mathrm{X}-100$, and blocked with $1 \%$ bovine serum albumin (BSA). They were then reacted with rhodamine phalloidin in PBS (1:50) for 20 min in the dark and mounted with mounting medium with DAPI (Vector Laboratories, Burlingame, CA, USA) to label the nuclei. The stained cells were observed using a confocal laser scanning microscope (Carl Zeiss, Oberkochen, Germany).

2.6. Alkaline Phosphatase (ALP) Staining. The expression of ALP of MSCs was observed using an Alkaline phosphatase kit (Sigma) according to the manufacturer's protocol. The cells were fixed in fixation solution and dyed with alkaline-dye mixture. The nuclei of cells were then labeled with Mayer's hematoxylin solution for $10 \mathrm{~min}$.

2.7. $\alpha$-Smooth Muscle ( $\alpha$-SMA) Staining. Immunofluorescence staining was carried out to observe the expression of 
(a)

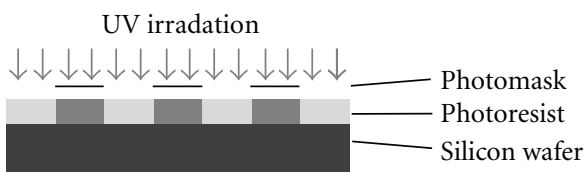

(b)

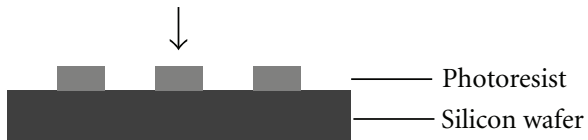

(c)

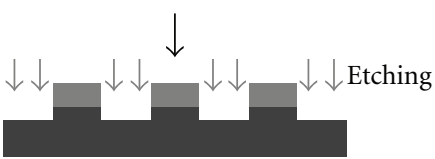

(d)

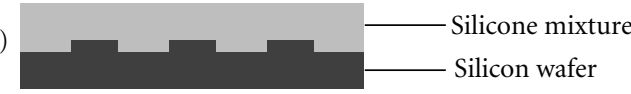

(e)

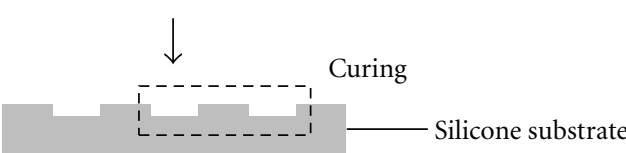

(A)

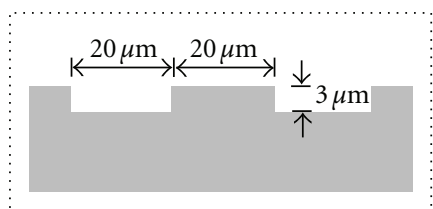

(B)
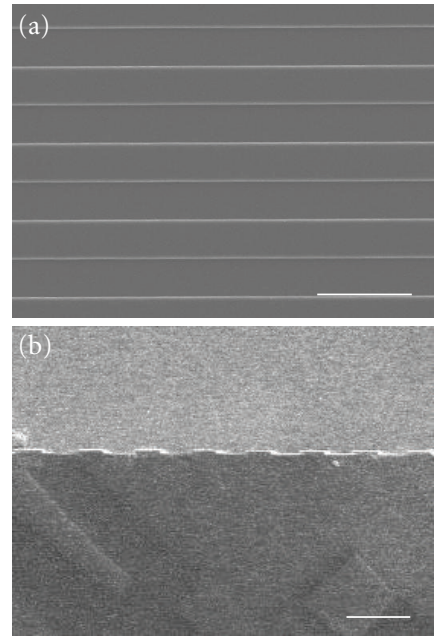

(C)

FIgURE 1: Fabrication of microgrooved substrates. (A) Procedure of fabrication of microgrooved substrates: (a) the negative photoresist was spin-coated onto a silicon wafer, exposed to UV, and polymerased selectively through photomask. (b) The unpolymerased photoresist was washed out. (c) A silicon wafer was etched through the process of DRIE. (d) Silicone mixture was poured onto the microgrooved silicon wafer, spin-coated, and cured at $125^{\circ} \mathrm{C}$ for $20 \mathrm{~min}$. (e) The microgrooved substrate was separated from the silicon wafer. (B) The dimensions of each groove were confirmed to be $20 \mu \mathrm{m}$ and $3 \mu \mathrm{m}$ for width and depth, respectively. (C) SEM images of microgrooved substrates: (a) top view and (b) cross section. (scale bar $=50 \mu \mathrm{m}$ ).

$\alpha$-SMA, a marker related to smooth muscle. The cells were fixed in $10 \%$ formalin solution, neutral buffered (Sigma), and incubated with $7.5 \%$ BSA to reduce nonspecific background staining. They were then incubated with $\alpha$-SMA primary antibody (Monoclonal Anti-Actin, $\alpha$ Smooth Muscle, $1: 200$, Sigma) for $1 \mathrm{~h}$, followed by incubation with fluorescein isothiocyanate- (FITC-)conjugated secondary antibody (Anti-mouse IgG FITC conjugate, 1:200, Sigma) for $1 \mathrm{~h}$. Fluorescence imaging was performed using a confocal laser scanning microscope (Carl Zeiss) and manager software.

2.8. Reverse Transcription-Polymerase Chain Reaction (RT$P C R$ ). RT-PCR was conducted to detect the expression of bone-related genes (OPN, BMP2), smooth muscle-related genes $(\alpha$-SMA, CDM), and GAPDH in the MSCs. GAPDH was used as a housekeeping gene. Total RNA was extracted using the RNeasy mini kit (Qiagen, Valencia, CA, USA), and cDNA was synthesized using the Omniscript RT kit (Qiagen) with an oligo(dT) primer. PCR was carried out to amplify the cDNA for a gene-specific number of cycles under the following conditions: $5 \mathrm{~min}$ incubation at $94^{\circ} \mathrm{C}$, $30 \mathrm{sec}$ denaturating at $94^{\circ} \mathrm{C}, 30 \mathrm{sec}$ annealing at the primerspecific temperature, and $40 \mathrm{sec}$ extension at $72^{\circ} \mathrm{C}$, followed by a 7 -min final extension step at $72^{\circ} \mathrm{C}$ (Table 1 ). After electrophoresis, the PCR products were visualized by ethidium bromide staining. The image densities of the PCR products were captured using a Low-Light Image System (GelDoc 2000; Bio-Rad, Hercules, CA, USA).

2.9. Statistical Analysis. The data are expressed as the mean \pm SD. Statistical analysis was performed using a two-way analysis of variance (ANOVA). When the ANOVA indicated a significant difference $(P \leq .05)$ between groups, the difference was evaluated using the least significant difference (LSD).

\section{Results}

3.1. DNA Content. The proliferation data for all groups are presented in Figure 2. In the absence of mechanical stimuli, MSCs increased gradually over time. Immediately following mechanical stimuli, the proliferation of MSCs was highly increased regardless of the magnitude of the stimuli or the microgrooves and remained high after the stimuli stopped.

3.2. Staining of Actin Filaments. Without stimuli, MSCs on the flat surface were oriented randomly (Figures 3(a), 3(g)), whereas those on the microgrooved surface were oriented along the direction of grooves (Figures $3(d), 3(j)$ ). In the presence of stimuli, MSCs on the flat surface tended to be oriented perpendicular to the direction of strain regardless 
TABLE 1: Sequence of PCR primers and product sizes. $\alpha$-SMA: $\alpha$-smooth muscle actin, CDM : caldesmon, OPN : osteopontin, BMP2 : bone morphogenic protein-2.

\begin{tabular}{|c|c|c|c|}
\hline Primer & Forward $(F)$ and reverse $(\mathrm{R}) \operatorname{primer}\left(5^{\prime}-3^{\prime}\right)$ & GeneBank accession no. & Product size (bp) \\
\hline \multirow[t]{2}{*}{$\alpha$-SMA } & (F) gatgaagcgcagagcaaaag & X60732 & 231 \\
\hline & (R) catggctgggacattgaaag & & \\
\hline \multirow[t]{2}{*}{$\mathrm{CDM}$} & (F) agaggcgatgggagaagaga & AF421381 & 131 \\
\hline & (R) tttcatcacgagcaacacca & & \\
\hline \multirow[t]{2}{*}{ OPN } & (F) ctccaatgaatccgacgatg & D16544 & 388 \\
\hline & (R) cacctggcttacatcatggc & & \\
\hline \multirow[t]{2}{*}{ BMP2 } & (F) cgcctcaaatccagctgtaag & AF041421 & 79 \\
\hline & (R) gggccacaatccagtcgtt & & \\
\hline \multirow[t]{2}{*}{ GAPDH } & (F) gtcgtctcctgcgacttcaa & NM_001082253 & 116 \\
\hline & (R) ccaccaccctgttgctgtag & & \\
\hline
\end{tabular}

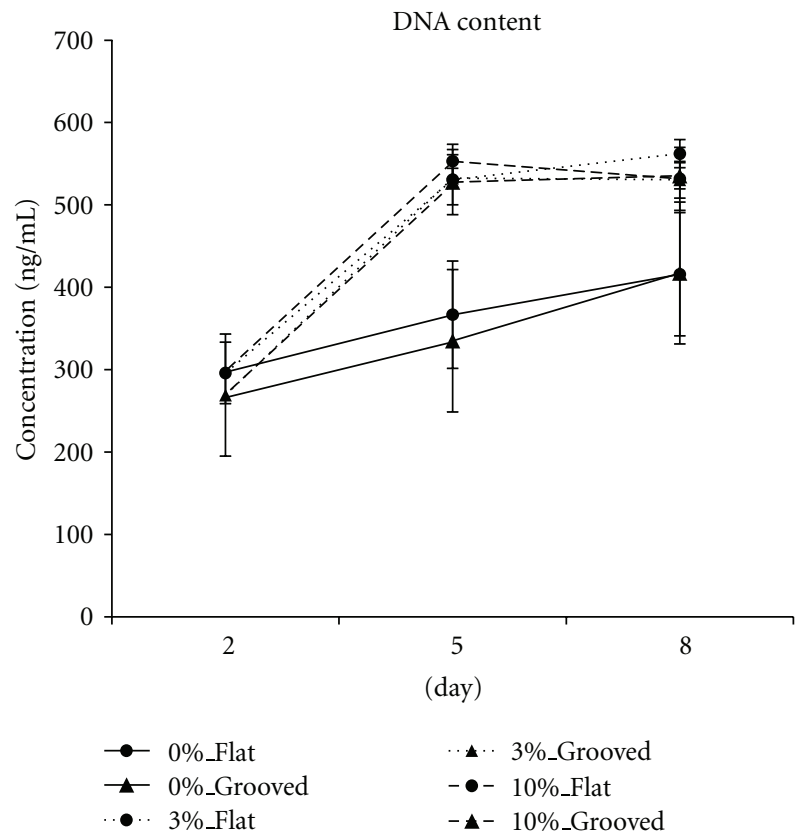

FIGURE 2: DNA contents of each group. The proliferation of strained MSCs was increased compared to that of unstrained MSCs regardless of the magnitudes of the stimuli or microgrooves. Mechanical stimuli were applied to MSCs for 3 days from $48 \mathrm{~h}$ after seeding (Day 2). Days 5 and 8 refer to the time points immediately after stimuli ceased and 3 days after stimuli ceased, respectively. $(n=5)$.

of the magnitude of the stimuli (Figures 3(b), 3(c), 3(h), 3(i)). The cells on the grooved surface also tended to reorient. However, the grooves acted as obstacles for cell reorientation, resulting in cellular arrangement of approximately 30-40 from the direction of the grooves (Figures 3(e), 3(f), 3(k), $3(1))$.
3.3. Staining of ALP and $\alpha$-SMA. The expression of ALP was observable on day 5 when $3 \%$ strain was imposed on cells on the flat surface (Figure 4(A)-(b)). No remarkable differences were found among the other groups (Figure $4(\mathrm{~A})$ ). The immunofluorescence images of MSCs showed that the expression of $\alpha$-SMA of MSCs cultured on microgrooved surfaces were generally enhanced compared to those cultured on flat surfaces (Figure 4(B)). In particular, the 10\% strain on microgrooved substrates induced substantial expression of $\alpha$-SMA (Figure 4(B)-(f), (1)).

3.4. RT-PCR. Figure 5(a) depicts the typical RT-PCR results for each group. The expression of each gene was normalized based on the expression of glyceraldehyde-3-phosphate dehydrogenase $(\mathrm{GAPDH})$, and results are presented in Figures 5(b) and 5(c). The expressions of $\alpha$-SMA and caldesmon (CDM), which are known to be early and intermediate markers of differentiation into SMCs, respectively, are shown in Figure 5(b). The expressions of $\alpha$-SMA and CDM on the grooved surfaces were observable compared to those on flat surfaces when strained. A significant difference in the expression of $\alpha$-SMA, the early marker, was found under 3\% strain and the difference was maintained until day 8 . The straining obviously increased the expression of CDM (day 5), and its effect was to be maintained until day 8 . However, the expression of CDM in $10 \%$ _Flat on day 8 was significantly decreased while its expression in 10\%_Grooved on the same day was maintained.

Even without straining, the flat surface enabled significantly higher expression of OPN than the grooved surface did on day 5 regardless of straining. This tendency continued even after straining was stopped on the flat surface. The straining effects on the expression of BMP2 were observed on day 5. The higher expressions of BMP2 were observed among the strained groups, even though they were not significant among the strained groups. The surface geometries did not show any effects on the expressions of BMP2 when MSCs 


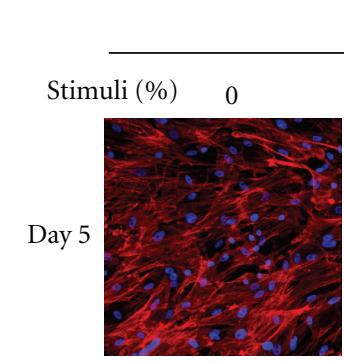

(a)

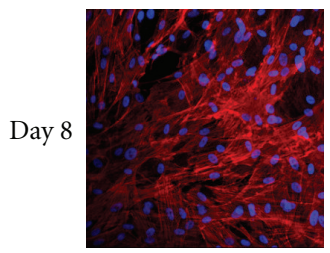

(g)

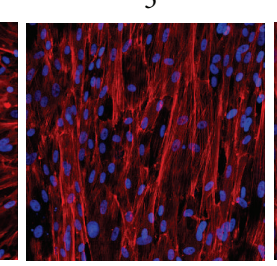

(b)

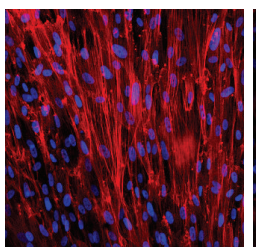

(h)
Flat

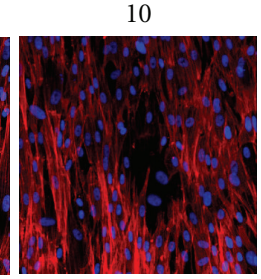

(c)

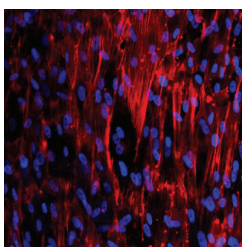

(i)

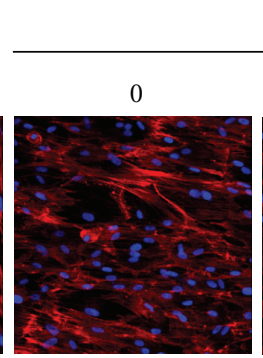

(d)

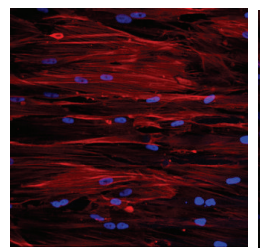

(j)
Microgrooved

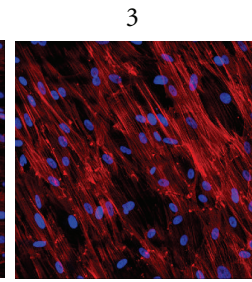

(e)

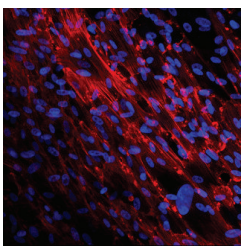

(k)
10

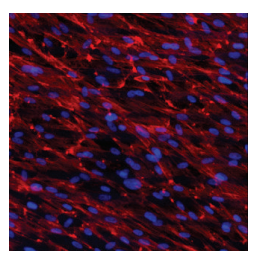

(f)

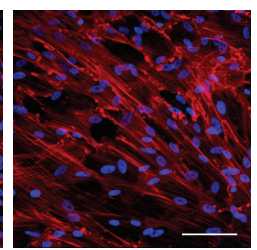

(1)

Direction of stimuli $\leftrightarrow /$ direction of microgrooves $\leftrightarrow$

FIgURE 3: Actin filaments of MSCs. On the flat surface, unstrained MSCs were randomly oriented (a) and strained MSCs were oriented perpendicular to the direction of grooves regardless of the magnitude of stimuli ((b), (c)). On the microgrooved surface, MSCs were oriented along the microgrooves without stimuli (d). Immediately following mechanical strain, MSCs were aligned away from the microgrooves ((e), (f)). The tendency of each group was maintained until 3 days after stimuli stopped ((g)-(l)). (All photographs were taken at the same magnification level; scale bar $=100 \mu \mathrm{m})$.

were strained. The expression patterns were maintained even though the straining was stopped (day 8).

\section{Discussion}

We used mechanical straining and patterns of substrates to differentiate MSCs without using biochemical reagents. Two different magnitudes of straining, $3 \%$ and $10 \%$, were applied for 3 days at a frequency of $0.26 \mathrm{~Hz}$ on cells seeded on flat or microgrooved surfaces.

Higher proliferation rates were observed when MSCs were strained regardless of the surface pattern. Therefore, physical straining is thought to enhance the proliferation of MSCs [15]. The groups without straining showed steady increases in proliferation. However, the strained groups showed increased proliferation on day 5 , and the increased proliferation rates were maintained until day 8 . This may suggest that the groups under mechanical straining started differentiating on day 8.

The confocal microscopic observation of actin filament arrangements showed that MSCs on the flat surface tended to rearrange perpendicular to the direction of straining, whereas those on the grooved surface tended shift to approximately $30-40^{\circ}$ away from the direction of the grooves. Kurpinski et al. [18] also reported such a perpendicular rearrangement of strained cells on a flat surface. Furthermore, they reported conflicting results for the arrangement of cells on a grooved surface (width $\sim 10 \mu \mathrm{m}$ ). This may be due to the use of narrower grooves, which may not have provided any marginal space, thus preventing the cells from rearranging.

Our results can be analyzed based the effects of straining magnitude and surface morphology on the differentiation of
MSCs. We used two different magnitudes of straining (3\% and $10 \%$ ) to investigate the effects of straining magnitude on the differentiation of MSCs. Previous research suggested that lower magnitudes of straining tended to induce osteogenesis, whereas relatively high-magnitude straining tended to induce differentiation of stem cells into SMCs or ligament cells $[10,12,13,19-22]$. Friedl et al. [19] reported the expression of osteo-chondrogenic genes when MSCs were cultured under $0.3 \%$ stretching conditions of $1 \mathrm{~Hz}$ for 3 days. In addition, the differentiation of MSCs into SMCs was reported by Nieponice et al. [13] when the cells were cultured under $10 \%$ straining at $1 \mathrm{~Hz}$ for 6 days.

Our ALP and $\alpha$-SMA staining analysis also showed comparable results to previous reports, as did the quantitative analyses of genes related to specific cell types.

The expression of $\alpha$-SMA decreased under 3\% straining and increased under $10 \%$ straining when cells were on flat surfaces. This suggests that more intense straining within a certain range had a positive effect on the early differentiation of MSCs into SMCs. This is supported by the report that the SMCs in blood vessels experience approximately 9$12 \%$ straining during normal physiological conditions in vivo [23]. Also, MSCs on the grooved surfaces showed higher expressions of $\alpha$-SMA than on the flat surfaces. The combined effects were confirmed through the two-way ANOVA test $(P<.001$, Data were not shown.). This may be because the grooves prevented the cells from returning to their original arrangement; thus, the cells maintained $\alpha$ SMA expression even after the straining was stopped. This hypothesis is supported by previous reports [14].

The effect of straining on the expression of CDM was observable on day 5 regardless of surface geometry. However, 


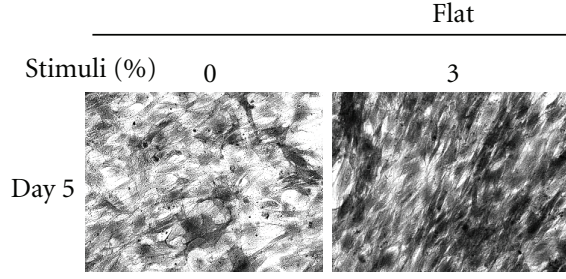

(a)

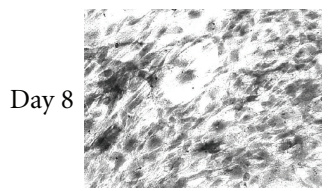

(g)

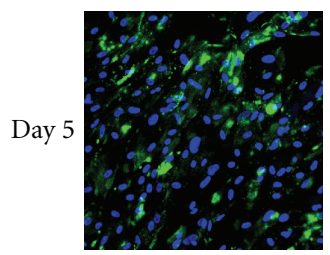

(a)

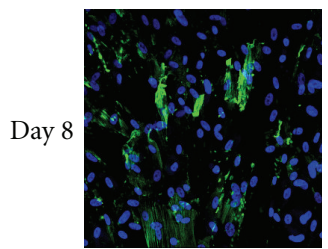

(g) (b)

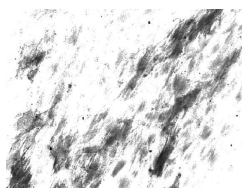

(h)

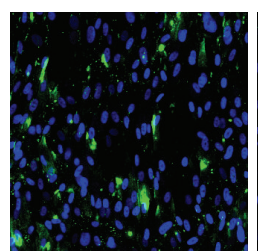

(b)

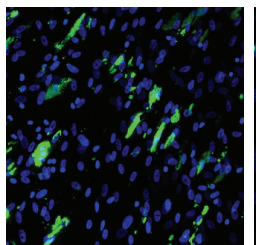

(h)

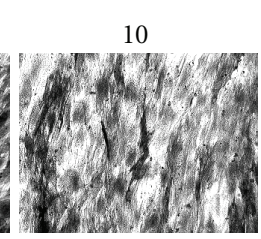

(c)

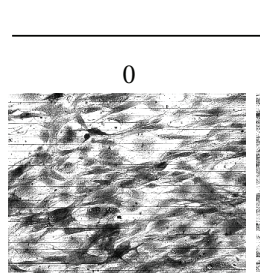

(d)

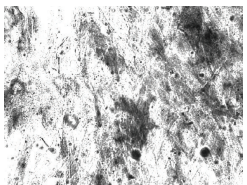

(i)

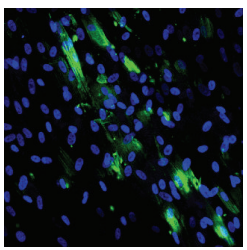

(c)

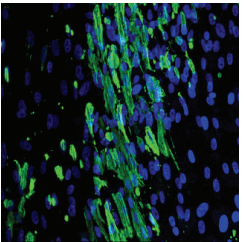

(i)

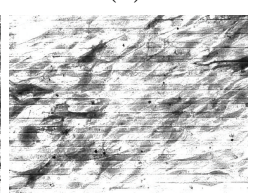

(j)

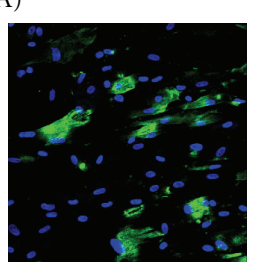

(d)

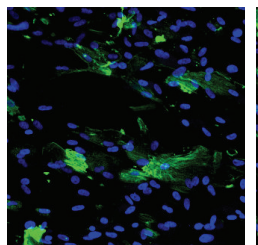

(j)
Microgrooved

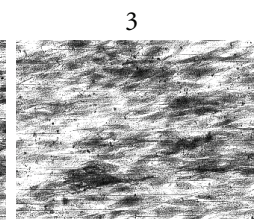

(e)

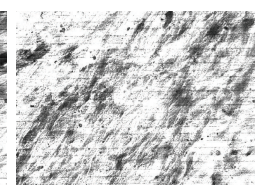

(k)

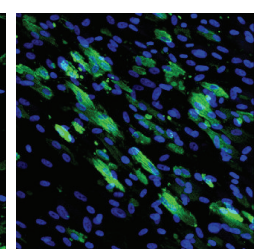

(e)

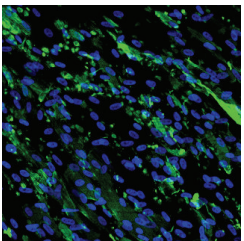

(k)
10

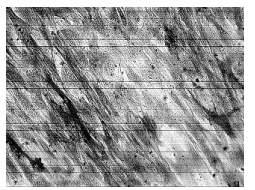

(f)

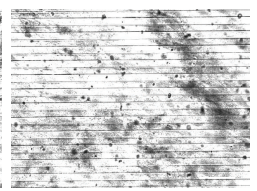

(1)

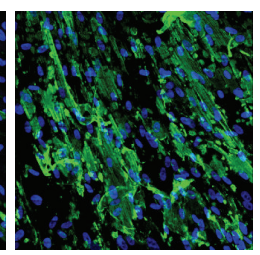

(f)

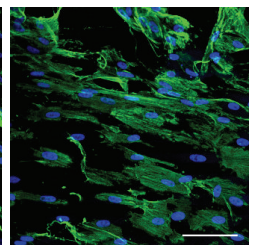

(1)

Direction of stimuli $\leftrightarrow /$ direction of microgrooves $\leftrightarrow$

(B)

Figure 4: Staining of ALP and $\alpha$-SMA. The expressions of ALP and $\alpha$-SMA in MSCs on day 5 ((a)-(f)) and day 8 ((g)-(l)) are presented in (A) and (B), respectively. The expression of ALP was substantial on day 5 when $3 \%$ of straining was imposed on the flat surface ((A)(b)). The expression of $\alpha$-SMA on the microgrooved surface was enhanced more than on the flat surface. In particular, the highest expression of $\alpha$-SMA was observed when MSCs were under $10 \%$ strain on the microgrooved surface. (All photographs were taken at same magnification level; scale bar $=100 \mu \mathrm{m}$ ).

dramatic decrease was observed on day 8 , that is, 3 days after straining was stopped in $10 \%$ _Flat, while the expression was maintained in 10\%_Grooved. This tendency is different from that of $\alpha$-SMA. $\alpha$-SMA and CDM are known to be one of the early and intermediate markers in relation to the differentiation of MSCs into SMC-like cell type, respectively [24]. Therefore, this different observation suggests us that we need to provide longer duration of straining to keep the comparable expression of CDM when MSCs are under higher strain and on flat surface. However, the CDM expression was maintained when MSCs were on the grooved surface (day 8 ), which explains that the surface geometry helps keep the expression of CDM. The results obtained from the expressions of $\alpha$-SMA and CDM suggest that both of higher straining and grooved surface are needed for the differentiation of MSCs into SMC-like cell type.

OPN and BMP2 are markers indicating the differentiation of stem cells into osteoblasts and are believed to play important roles in bone generation $[25,26]$. The expressions of OPN were more observable when MSCs were on the flat surfaces rather than on grooved surfaces regardless of straining (day 5). Among groups on the grooved surface, $3 \%$ straining showed significantly higher expression of OPN than the others. Significant decrease in the expression of OPN was observed when MSCs were under higher strain $(10 \%)$ on grooved surfaces (day 5,8$)$. This result suggests that higher straining tends to suppress the expression of OPN on the grooved surface. Even after the straining was stopped higher expressions of OPN were observed and maintained provided that lower strain was engaged regardless of surface geometry.

The overall expression of BMP2 was enhanced when strained; however, the magnitude of straining did not affect the degree of expression. Also, the effect of straining was maintained even after the stimulation was stopped (day 8). This indicates that the straining contributes to the expression of BMP2 regardless of surface geometry. These results suggest that the combining conditions of lower strain and flat surface 


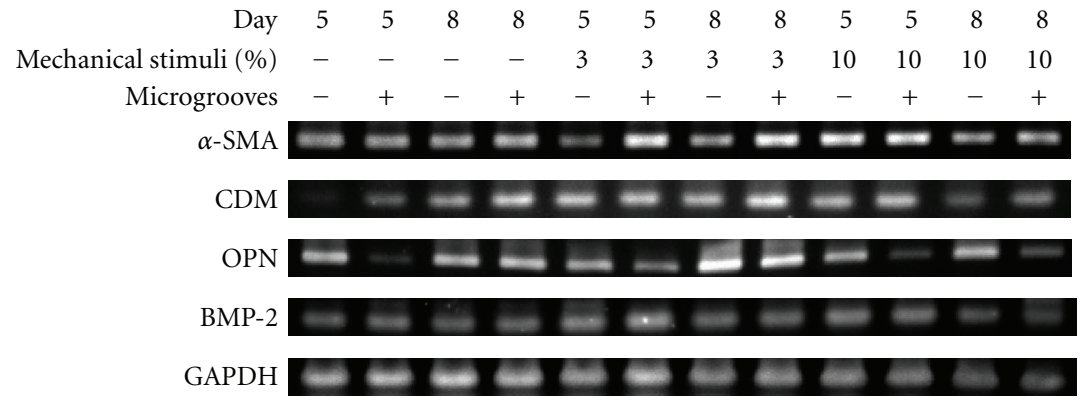

(a)

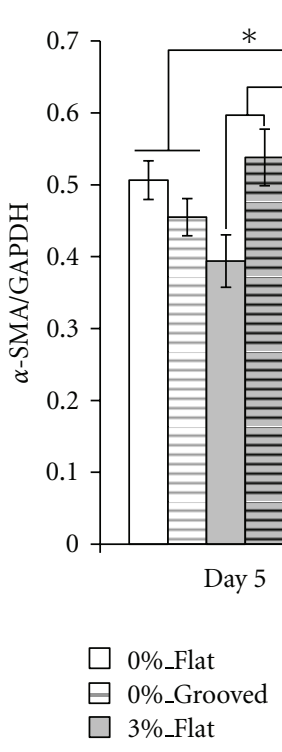

$\alpha$-SMA

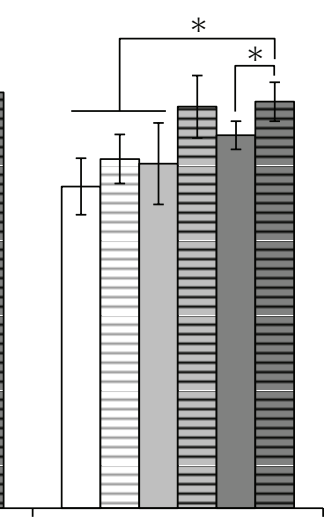

Day 8

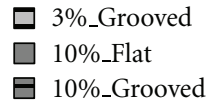

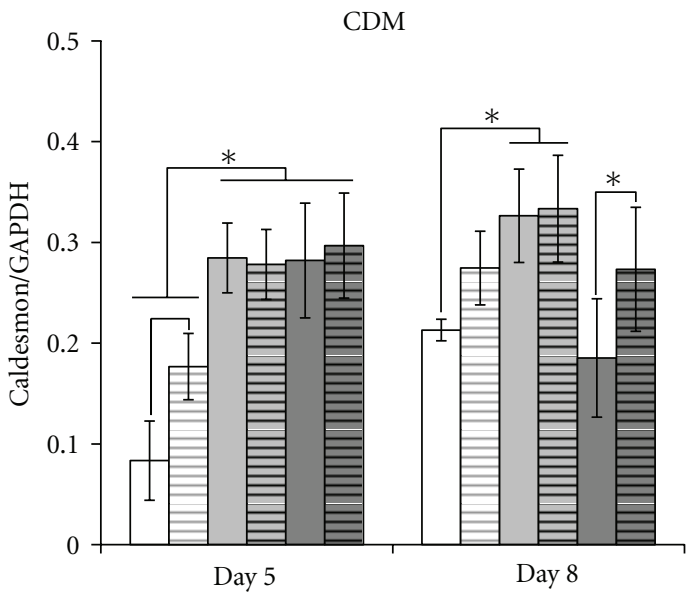
$\square$ 0\%_Flat
日 $0 \%$ _Grooved
$\square$ 3\%_Flat

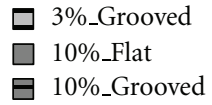

(b)
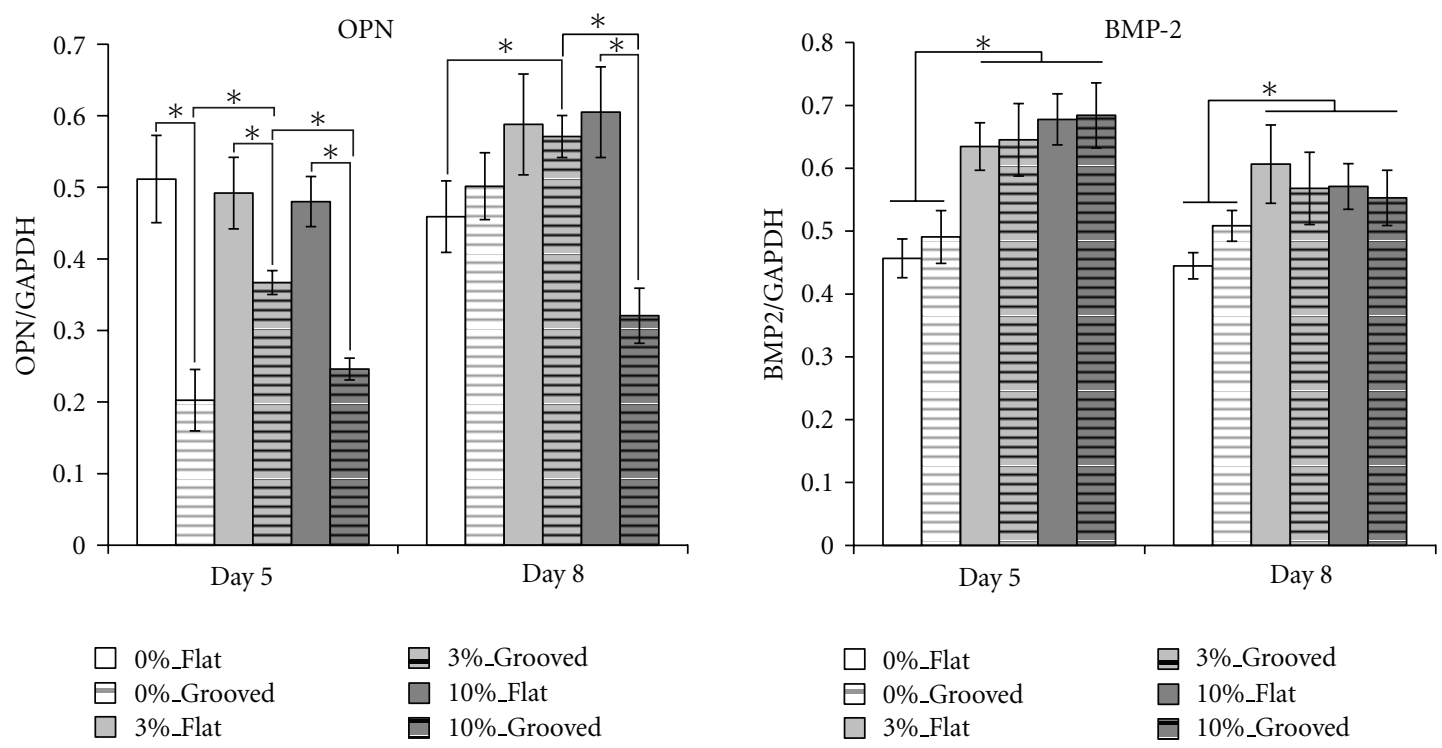

(c)

FigURE 5: Gene expression of MSCs. (a) Gene expression profiles for each marker with/without mechanical stimuli or microgrooves. The expression levels of each gene are normalized by the expression of GAPDH; (b) smooth muscle cell-related genes; (c) osteoblast-related genes. Days 5 and 8 represent the time points immediately after stimuli ceased and 3 days after stimuli ceased, respectively. (All data are represented as mean \pm SD. $n=3,{ }^{*}$ denotes $P \leq .05$.) 
can contribute to the expressions of OPN and BMP2, which are known to be the markers indicating osteoblast-like cell type.

\section{Conclusions}

We aimed to investigate the effects of biomechanical straining and surface patterns of substrates on MSCs without using any of the typical biochemical reagents that promote MSC proliferation or differentiation. Given our results, the following conclusions can be drawn: (i) mechanical straining enhances the proliferation of MSCs regardless of surface patterns; (ii) lower level straining (3\% in this study) positively effects the differentiation of MSCs into osteo-related cells; (iii) higher level straining (10\% in this study) positively influences the differentiation of MSCs into smooth muscle-like cells; (iv) a grooved surface provides a preferable environment for MSC differentiation into smooth muscle-like cells, whereas flat surfaces are more effective in promoting differentiation into osteo-related cells.

Our study confirms that controlling microenvironments so that they mimic those in vivo contributes to the differentiation of MSCs into specific cell types even without the use of biochemical reagents. However, the experimental conditions of this study, such as only $3 \%$ and $10 \%$ straining, the fixed geometry of the surface grooves, and a fixed straining frequency $(0.26 \mathrm{~Hz})$ were limited, and a wider variety of parameters should be adopted for future studies.

\section{Acknowledgment}

This work was supported by the National Research Foundation National Research Lab (Grant No. 2010-0018435).

\section{References}

[1] F. P. Barry and J. M. Murphy, "Mesenchymal stem cells: clinical applications and biological characterization," International Journal of Biochemistry and Cell Biology, vol. 36, no. 4, pp. 568$584,2004$.

[2] R. J. Deans and A. B. Moseley, "Mesenchymal stem cells: biology and potential clinical uses," Experimental Hematology, vol. 28, no. 8, pp. 875-884, 2000.

[3] M. F. Pittenger, A. M. Mackay, S. C. Beck et al., "Multilineage potential of adult human mesenchymal stem cells," Science, vol. 284, no. 5411, pp. 143-147, 1999.

[4] W. J. Li, R. Tuli, X. Huang, P. Laquerriere, and R. S. Tuan, "Multilineage differentiation of human mesenchymal stem cells in a three-dimensional nanofibrous scaffold," Biomaterials, vol. 26, no. 25, pp. 5158-5166, 2005.

[5] D. Wang, J. S. Park, J. S. F. Chu et al., "Proteomic profiling of bone marrow mesenchymal stem cells upon transforming growth factor $\beta 1$ stimulation," The Journal of Biological Chemistry, vol. 279, no. 42, pp. 43725-43734, 2004.

[6] M. Skobe, T. Hawighorst, D. G. Jackson et al., "Induction of tumor lymphangiogenesis by VEGF-C promotes breast cancer metastasis," Nature Medicine, vol. 7, no. 2, pp. 192-198, 2001.

[7] A. J. Engler, S. Sen, H. L. Sweeney, and D. E. Discher, "Matrix Elasticity Directs Stem Cell Lineage Specification," Cell, vol. 126, no. 4, pp. 677-689, 2006.
[8] S. J. Gwak, S. H. Bhang, I. K. Kim et al., "The effect of cyclic strain on embryonic stem cell-derived cardiomyocytes," Biomaterials, vol. 29, no. 7, pp. 844-856, 2008.

[9] A. Ignatius, H. Blessing, A. Liedert et al., "Tissue engineering of bone: effects of mechanical strain on osteoblastic cells in type I collagen matrices," Biomaterials, vol. 26, no. 3, pp. 311318, 2005.

[10] W. C. C. Lee, T. M. Maul, D. A. Vorp, J. P. Rubin, and K. G. Marra, "Effects of uniaxial cyclic strain on adipose-derived stem cell morphology, proliferation, and differentiation," Biomechanics and Modeling in Mechanobiology, vol. 6, no. 4, pp. 265-273, 2007.

[11] R. D. Sumanasinghe, S. H. Bernacki, and E. G. Loboa, "Osteogenic differentiation of human mesenchymal stem cells in collagen matrices: effect of uniaxial cyclic tensile strain on bone morphogenetic protein (BMP2) mRNA expression," Tissue Engineering, vol. 12, no. 12, pp. 3459-3465, 2006.

[12] Y. J. Chen, C. H. Huang, I. C. Lee, Y. T. Lee, M. H. Chen, and T. H. Young, "Effects of cyclic mechanical stretching on the mRNA expression of tendon/ ligament-related and osteoblastspecific genes in human mesenchymal stem cells," Connective Tissue Research, vol. 49, no. 1, pp. 7-14, 2008.

[13] A. Nieponice, T. M. Maul, J. M. Cumer, L. Soletti, and D. A. Vorp, "Mechanical stimulation induces morphological and phenotypic changes in bone marrow-derived progenitor cells within a three-dimensional fibrin matrix," Journal of Biomedical Materials Research-Part A, vol. 81, no. 3, pp. 523530, 2007.

[14] J. S. Park, J. S. F. Chu, C. Cheng, F. Chen, D. Chen, and S. Li, "Differential effects of equiaxial and uniaxial strain on mesenchymal stem cells," Biotechnology and Bioengineering, vol. 88, no. 3, pp. 359-368, 2004.

[15] G. Song, Y. Ju, X. Shen, Q. Luo, Y. Shi, and J. Qin, "Mechanical stretch promotes proliferation of rat bone marrow mesenchymal stem cells," Colloids and Surfaces B, vol. 58, no. 2, pp. 271$277,2007$.

[16] C. H. Lee, H. J. Shin, I. H. Cho et al., "Nanofiber alignment and direction of mechanical strain affect the ECM production of human ACL fibroblast," Biomaterials, vol. 26, no. 11, pp. 1261-1270, 2005.

[17] D. H. Kim, S. H. Kim, S. J. Heo et al., "Enhanced differentiation of mesenchymal stem cells into NP-like cells via 3D coculturing with mechanical stimulation," Journal of Bioscience and Bioengineering, vol. 108, no. 1, pp. 63-67, 2009.

[18] K. Kurpinski, J. Chu, C. Hashi, and S. Li, "Anisotropic mechanosensing by mesenchymal stem cells," Proceedings of the National Academy of Sciences of the United States of America, vol. 103, no. 44, pp. 16095-16100, 2006.

[19] G. Friedl, H. Schmidt, I. Rehak, G. Kostner, K. Schauenstein, and R. Windhager, "Undifferentiated human mesenchymal stem cells (hMSCs) are highly sensitive to mechanical strain: transcriptionally controlled early osteo-chondrogenic response in vitro," Osteoarthritis and Cartilage, vol. 15, no. 11, pp. 1293-1300, 2007.

[20] M. C. Qi, J. Hu, S. J. Zou, H. Q. Chen, H. X. Zhou, and L. C. Han, "Mechanical strain induces osteogenic differentiation: Cbfa1 and Ets-1 expression in stretched rat mesenchymal stem cells," International Journal of Oral and Maxillofacial Surgery, vol. 37, no. 5, pp. 453-458, 2008.

[21] G. M. Riha, X. Wang, H. Wang et al., "Cyclic strain induces vascular smooth muscle cell differentiation from murine embryonic mesenchymal progenitor cells," Surgery, vol. 141, no. 3, pp. 394-402, 2007. 
[22] D. F. Ward, R. M. Salasznyk, R. F. Klees et al., "Mechanical strain enhances extracellular matrix-induced gene focusing and promotes osteogenic differentiation of human mesenchymal stem cells through an extracellular-related kinasedependent pathway," Stem Cells and Development, vol. 16, no. 3, pp. 467-479, 2007.

[23] G. B. Chapman, W. Durante, J. D. Hellums, and A. I. Schafer, "Physiological cyclic stretch causes cell cycle arrest in cultured vascular smooth muscle cells," American Journal of Physiology, vol. 278, no. 3, pp. H748-H754, 2000.

[24] S. G. Ball, A. C. Shuttleworth, and C. M. Kielty, "Direct cell contact influences bone marrow mesenchymal stem cell fate," International Journal of Biochemistry and Cell Biology, vol. 36, no. 4, pp. 714-727, 2004.

[25] D. Chen, M. Zhao, and G. R. Mundy, "Bone morphogenetic proteins," Growth Factors, vol. 22, no. 4, pp. 233-241, 2004.

[26] S. T. Choi, J. H. Kim, E. J. Kang et al., "Osteopontin might be involved in bone remodelling rather than in inflammation in ankylosing spondylitis," Rheumatology, vol. 47, no. 12, pp. 1775-1779, 2008. 

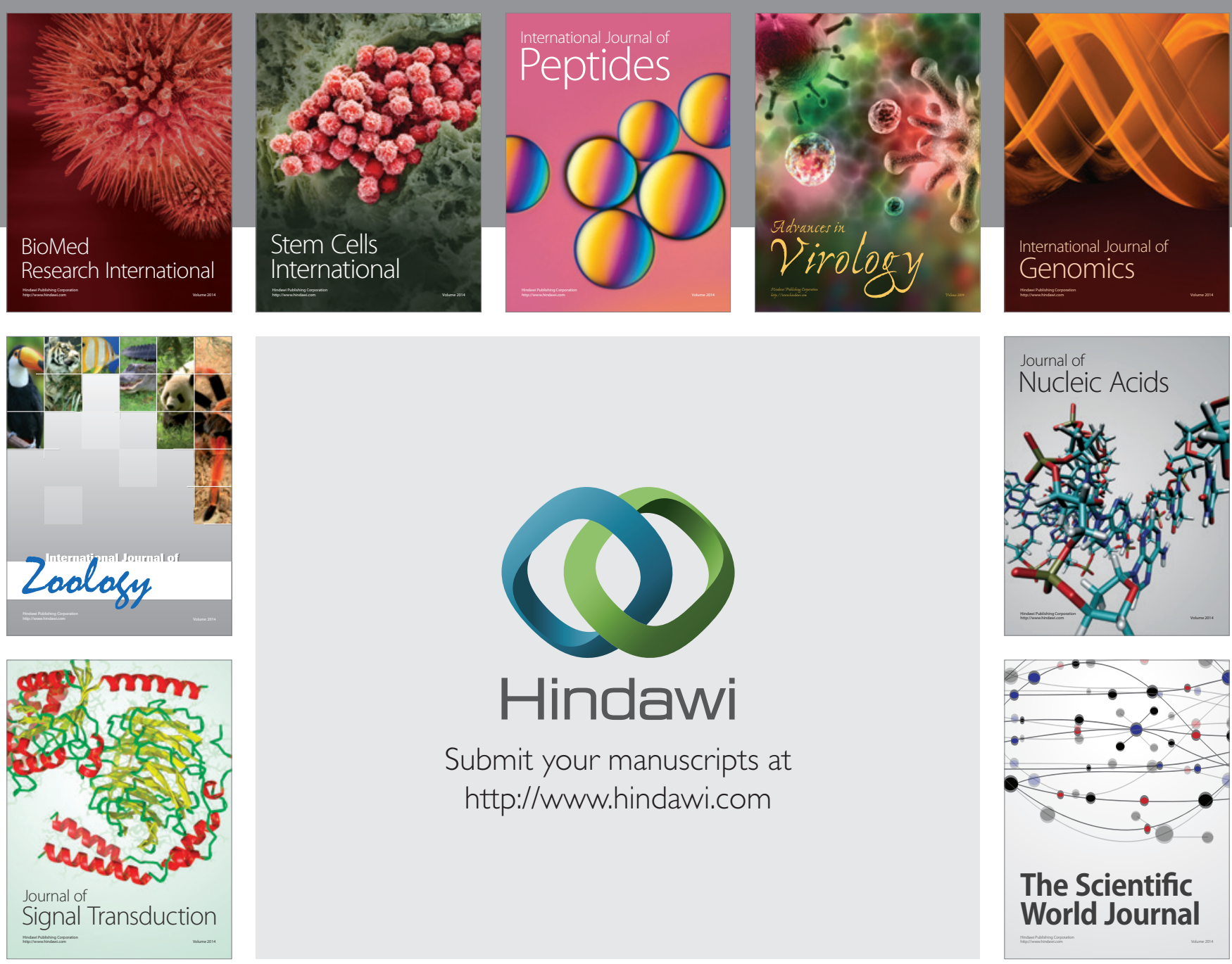

Submit your manuscripts at

http://www.hindawi.com
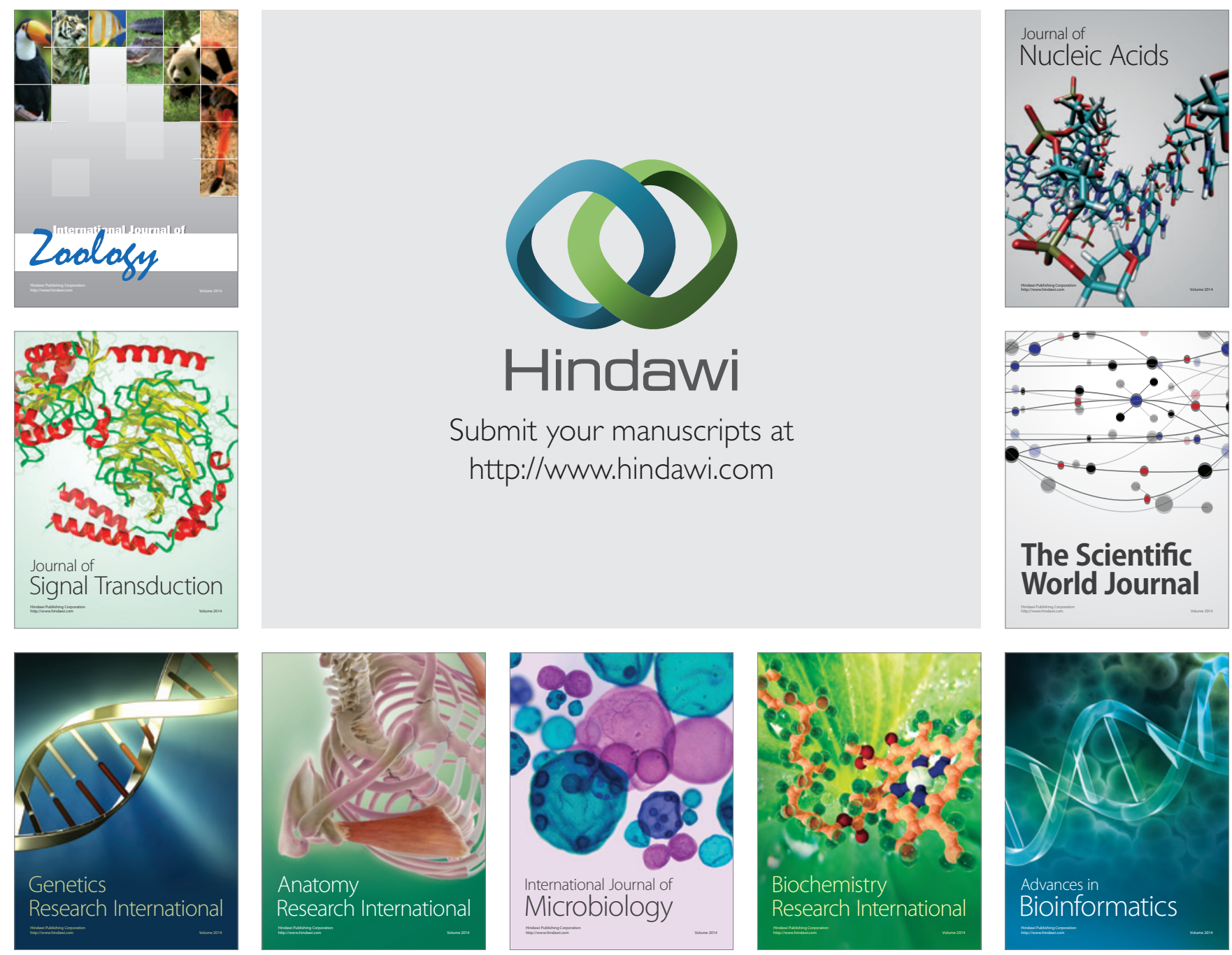

The Scientific World Journal
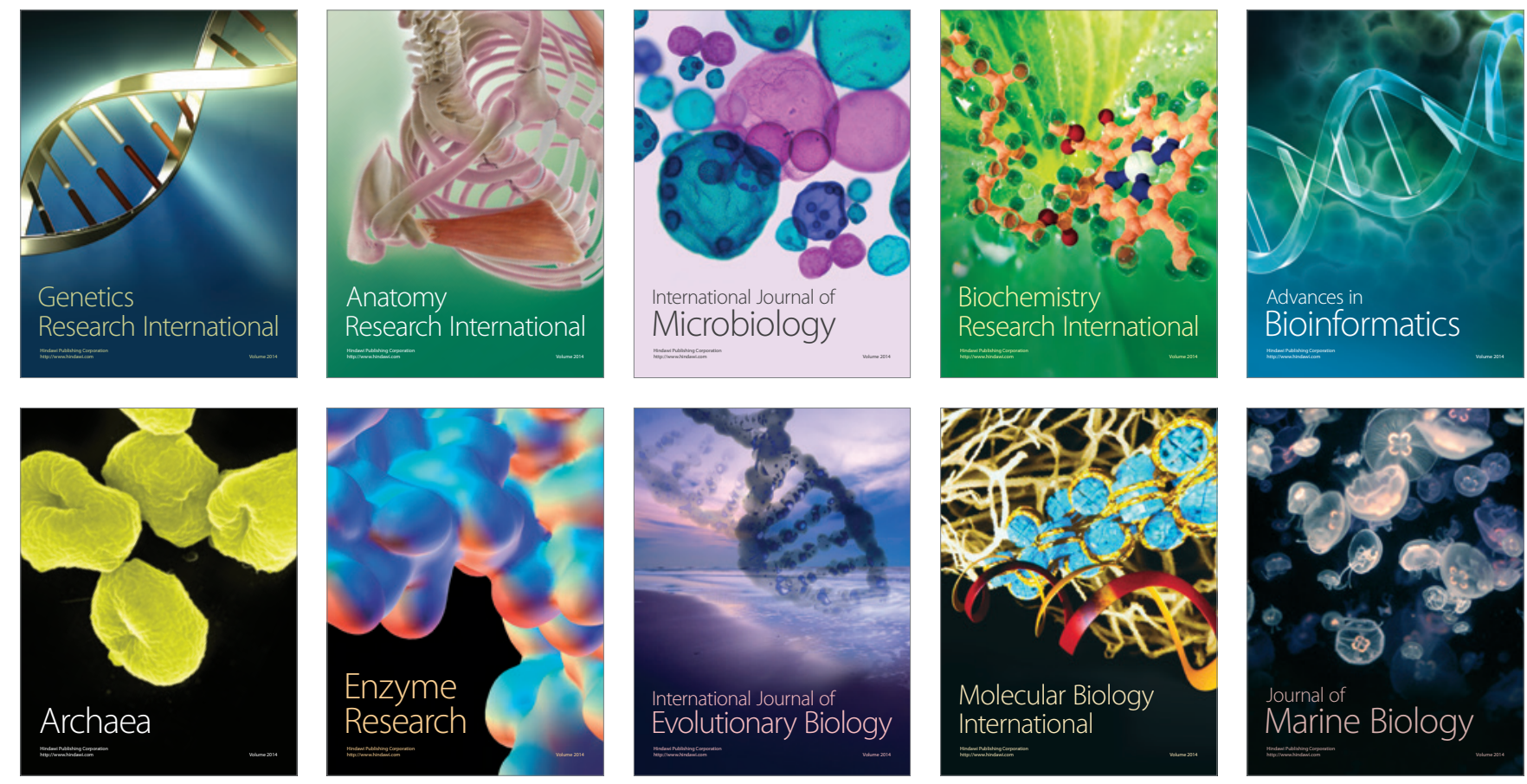